\title{
Essais
}

ESSAIS

Revue interdisciplinaire d'Humanités

$15 \mid 2019$

Jouer l'histoire

\section{Apprendre la Grande Guerre avec Soldats Inconnus? Mécaniques ludo-pédagogiques et introduction en milieu scolaire}

Romain Vincent

\section{CpenEdition}

Journals

Édition électronique

URL : http://journals.openedition.org/essais/1498

DOI : 10.4000/essais. 1498

ISSN : 2276-0970

Éditeur

École doctorale Montaigne Humanités

Édition imprimée

Date de publication : 15 octobre 2019

Pagination : 65-76

ISBN : 979-10-97024-07-9

ISSN : $2417-4211$

Référence électronique

Romain Vincent, «Apprendre la Grande Guerre avec Soldats Inconnus ? Mécaniques ludo-

pédagogiques et introduction en milieu scolaire », Essais [En ligne], 15 | 2019, mis en ligne le 05

octobre 2020, consulté le 12 octobre 2020. URL : http://journals.openedition.org/essais/1498 ; DOI :

https://doi.org/10.4000/essais.1498 


\title{
Apprendre la Grande Guerre avec Soldats Inconnus? \\ Mécaniques ludo-pédagogiques et introduction en milieu scolaire
}

\author{
Romain Vincent
}

«Un jeu qu'Ubisoft aimerait [...] aussi proposer comme outil pédagogique dans les écoles ${ }^{1}$. »

En conclusion de son article, Antoine Krempf dresse le lien possible entre un jeu de divertissement et son utilisation en classe. En effet, Soldats Inconnus : Mémoires de la Grande Guerre a tout du candidat idéal. Le jeu développé par Ubisoft en 2014 a connu un succès critique unanime grâce à une direction artistique soignée servant un gameplay original pour un jeu vidéo sur la Grande Guerre : "Soldats Inconnus n'est pas un jeu de guerre, mais un jeu sur la guerre » lit-on dans de nombreux articles, à la suite de Paul Tumélaire, directeur créatif ${ }^{2}$. Soldats Inconnus est un jeu d'aventure où le joueur incarne plusieurs personnages, militaires et civils, impliqués dans la Première Guerre mondiale. Ce jeu vidéo fait également partie d'un ensemble de productions plus larges labellisé par la Mission centenaire 14-183. Ainsi, nous proposons d'analyser Soldats Inconnus comme un dispositif ludique hybride entre un jeu vidéo de divertissement et un logiciel au but éducatif explicite, le faisant parfois se rapprocher d'un serious game, c'est-à-dire une œuvre qui a un autre objectif que le divertissement ${ }^{4}$. À la suite de Gilles Brougère, nous considérons

1 Antoine Krempf, "Soldats Inconnus, un jeu pour connaître la guerre ", Francetvinfo.fr, 25 juin 2014 : https://www.francetvinfo.fr/societe/soldats-inconnus-un-jeu-pour-connaitre-lagrande-guerre_1691677.html (dernière visite le 6 novembre 2018).

2 Antoine Flandrin, «Soldats Inconnus est un jeu vidéo sur la Grande Guerre mais ce n'est pas un jeu de guerre ", lemonde.fr, 19 mai 2014 : https://www.lemonde.fr/pixels/article/2014/05/19/ soldats-inconnus-est-un-jeu-video-sur-la-grande-guerre-mais-ce-n-est-pas-un-jeu-deguerre_4421313_4408996.html (dernière visite le 6 novembre 2018).

3 Mission centenaire 14-18, portail officiel du Centenaire de la Première Guerre mondiale : http://www.centenaire.org/fr.

4 Julian Alvarez, Djamien Djaouti et Olivier Rampnoux, Apprendre avec les serious games?, Futuroscope, Canopé Éditions, 2016. 
le jeu comme une activité autotélique, une pratique où le joueur se concentre sur l'activité elle-même et non sur ses conséquences supposées, ici, l'apprentissage ${ }^{5}$. Par conséquent, c'est précisément cette tension produite par la présence de ces deux objectifs au sein du même objet que nous souhaitons étudier.

Avec des jeux de gestion, comme la série Civilization, un jeu vidéo peut permettre d'aborder des savoirs procéduraux, comme des liens de causalité. À l'inverse, des savoirs d'ordre déclaratifs, comme des faits historiques sur la Première Guerre mondiale, semblent plus difficiles d'accès : s'il peut en intégrer dans son scénario, il paraît difficile d'affirmer que l'interaction produite par le joueur lui permette de les assimiler. Après une étude du dispositif pédagogique interne au jeu, nous désirons observer comment on joue à Soldats Inconnus dans le lieu d'apprentissage par excellence, l'école, en rendant compte de plusieurs pratiques enseignantes.

\section{Soldats Inconnus : Apprendre en jouant?}

La Première Guerre mondiale était généralement ludoformée dans des productions mettant en avant la violence du conflit avec des first-person shooter comme Necrovision ou le plus récent Battlefield 1. Soldats Inconnus propose une approche originale en s'intéressant à l'expérience combattante des différents personnages incarnés par le joueur (Figure 1). Émile est un paysan de Saint-Mihiel dans l'est de la France. Karl est un Allemand vivant lui aussi à Saint-Mihiel, marié à la fille d'Émile. Il doit cependant retourner en Allemagne, car il est appelé au front au début du jeu. Pour finir, Freddy est un Américain engagé volontaire, soulignant le caractère mondial de la guerre, tandis qu'Anna, l'infirmière belge, symbolise, en une seule personne, l'importance des femmes et des civils dans le conflit.
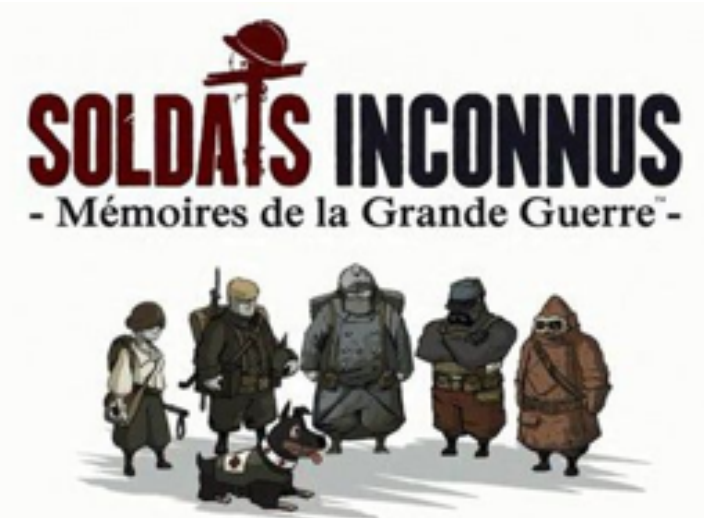

Figure 1 : Les différents personnages de Soldats Inconnus (C 2014, Ubisoft Entertainment)

5 Gilles Brougère, Jouer/apprendre, Paris, Economica, 2005. 
Au fil d'un scénario emmenant nos héros de 1914 à 1917, le joueur rencontre différents aspects du conflit : la conscription en 1914, les Gaz d'Ypres de 1915, le statut des prisonniers de guerre, l'expérience des tranchées de Verdun et de la Somme. Ainsi, le déroulement de Soldats Inconnus est rythmé par la grande Histoire et ne montre pas aux joueurs que des théâtres d'affrontements en le faisant notamment évoluer à l'arrière dans certains niveaux, comme lors du bombardement de la ville de Reims. Présenté ainsi, on serait tenté d'affirmer que Soldats Inconnus aborde le concept de " guerre totale ", désignant un conflit mobilisant toutes les ressources d'un état et impliquant civils et militaires dans un affrontement mondial.

Or, chacun des personnages incarnés par le joueur est animé par des motivations très personnelles. Freddy voulant venger la mort de sa femme lors d'un bombardement ou Anna désirant retrouver son père disparu pendant le conflit. Le patriotisme des soldats de 14-18 est ainsi évincé au profit de raisons plus consensuelles. Tout juste l'entraide des personnages, au cours de leurs multiples croisements pendant le scénario, nous permet d'aborder la camaraderie de tranchées, mais de manière très succincte. Si le jeu vidéo est un médium fondé sur l'interactivité qu'entretient le joueur avec le dispositif ludique, il contient également des instants de non-jeu. Dans l'introduction, la contextualisation du conflit se fait au prix de quelques torsions de la réalité pour faciliter la compréhension du joueur : le déclenchement du conflit est résumé à l'assassinat du prince d'Autriche-Hongrie, mettant ainsi de côté les nombreuses sources de tensions préexistant au premier conflit mondial, et la description du jeu des alliances de l'été 1914 omet certaines étapes ${ }^{6}$. De plus, l'introduction manipule certaines photographies d'archives (Figure 2). Le Jaurès historique (à gauche) photographié en pleine manifestation pacifique au Pré-Saint-Gervais en mai 1913 est traduit par les designers d'Ubisoft en un foudre de guerre anonyme, brandissant le drapeau tricolore et une affiche de mobilisation. Cette représentation du passé dépolitisée et aseptisée pourrait constituer un frein à son exploitation pédagogique. Ce sont justement par ces instants de non-jeu que Soldats Inconnus compte transmettre une partie de son contenu historique. Chaque chapitre du jeu est introduit par une scène cinématique, accompagnée d'une voix off nous donnant le cadre chronologique et géographique de la mission. De plus, au fil de sa progression, le joueur se voit proposer la lecture de fiches pédagogiques sur les thèmes rencontrés pendant le niveau. Au cours de celui se déroulant dans la ville d'Ypres, on peut en apprendre plus sur le fonctionnement des gaz de combat et leurs dégâts. Cette volonté de transmettre des faits se fait cependant au détriment de la dyna-

6 Extrait de l'introduction du jeu : "Suite à l'assassinat de François-Ferdinand, prince héritier de l'empire austro-hongrois, l'empire germanique déclare la guerre à la Russie ». Cette rapide explication omet les événements. 
mique ludique. Ces moments d'apprentissage apparaissent en plein milieu des niveaux et mettent entre parenthèses l'activité du joueur. La lecture est accompagnée d'une photographie d'époque, extraite du documentaire Apocalypse ${ }^{7}$, entraînant une rupture diégétique avec l'ambiance graphique du jeu (Figure 3).

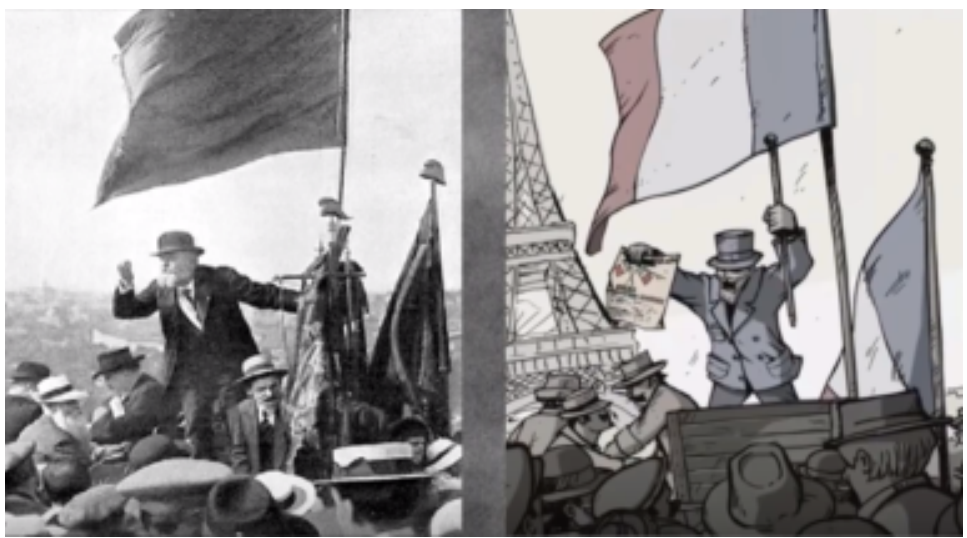

Figure 2 : À gauche : Jean Jaures au près Saint-Gervais, le 25 mai 1913

À droite : Extrait de l'introduction de Soldats Inconnus (C) 2014, Ubisoft Entertainment)

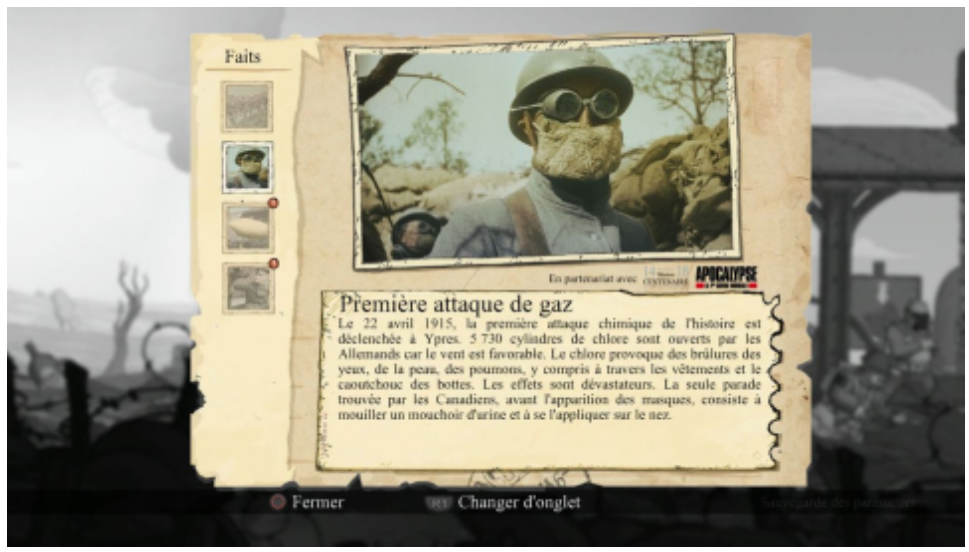

Figure 3 : Une fiche pédagogique proposée au joueur (C) 2014, Ubisoft Entertainment)

Par conséquent, Soldats Inconnus tend à séparer les dimensions sérieuses et ludiques, rendues encore plus visibles par la nature des énigmes très, voire trop, souvent détachées du contexte historique. En effet, elles se résument souvent par des puzzle-games qui n'ont que peu de lien avec la Grande Guerre. Le jeu propose également au joueur de collecter des objets du quotidien, comme de l'artisanat de tranchées, lettres de soldats, voire des brevets de marraines de guerre. Chaque instant de collecte est accompagné d'une fiche pédagogique

7 Isabelle Clarke, Daniel Costelle (réal.), (2009), Apocalypse : la seconde guerre mondiale, France Télévisions. 
expliquant les fonctions de l'objet. Ces éléments apportent une réelle plusvalue à la compréhension du quotidien des soldats et des civils, cependant cette quête est optionnelle : le joueur peut traverser toute l'aventure sans ne les avoir jamais consultés. Ces objets historiques ne sont pas intégrés aux énigmes et ne sont par conséquent jamais manipulés par le joueur, ce qui aurait pu être l'occasion pour lui de comprendre leur importance en jouant.

Dans Soldats Inconnus, le contenu factuel sur la Grande Guerre est porté au joueur par des scènes cinématiques au cours desquelles le joueur reste passif, ou par des items qui ne sont pas interactifs. En d'autres termes, Soldats Inconnus n'entraîne pas un apprentissage des notions de la Grande Guerre par des mécaniques ludiques. Nous rejoignons ainsi Jesper Juul qui estime que ces " éléments narratifs tendent [...] à aller à l'encontre du caractère proprement vidéoludique du jeu $»^{8}$.

\section{Jouer à Soldats Inconnus pour changer l'école?}

La sortie de Soldats Inconnus en 2014 rencontre un contexte politique et institutionnel globalement favorable à l'utilisation du numérique éducatif. Le Plan numérique pour l'éducation du quinquennat Hollande est accompagné d'une réflexion sur la forme scolaire, qui déboucha en mai 2017 sur la publication d'un rapport de l'Inspection Générale de l'Éducation nationale intitulé : La forme scolaire à l'heure du numérique?.

Chargée du rapport, Catherine Becchetti-Bizot, inspectrice générale de l'éducation nationale, dresse un tableau du modèle actuel de transmissions des savoirs à l'école. Ce document est organisé en trois parties : après avoir appelé le contexte actuel et les enjeux incombant au système scolaire, Becchetti-Bizot présente les nécessités de la transformation de la forme scolaire, et finit sur les initiatives innovantes déjà en cours sur le terrain qui pourraient servir comme modèle pour le reste de la communauté éducative.

Cette révolution des pratiques est jugée indispensable et incontournable : ainsi apprend-on que les "classes d'aujourd'hui ressemblent toujours à s'y méprendre à celle des écoles de la fin du XIX ${ }^{\mathrm{e}}$ siècle ${ }^{10}$. De fait, cet immobilisme du système éducatif ne permettrait pas de répondre aux défis actuels. Si des objectifs de bons sens sont avancés, comme " Répondre à la diversité et à l'hétérogénéité croissante des élèves ${ }^{11}$ ou " réduire les inégalités liées à un

8 Jesper Juul, «A clash between game and narrative », Digital Arts and Culture conference, Bergen, Norvège, 1998.

9 Catherine Becchetti-Bizot, Repenser la forme scolaire à l'heure du numérique. Vers de nouvelles manières d'apprendre et d'enseigner, Inspection générale de l'Éducation nationale, 2017.

10 Ibid., p. 1.

11 Ibid., p. 6. 
déterminisme social très lourd $»^{12}$, la nécessité du changement proviendrait plutôt de la transformation de la société : "Le numérique n'est pas seulement une révolution technologique [...] il est aussi un phénomène culturel et social » dans lequel l'École serait " prise dans le mouvement".

Dans cette optique de changement, le jeu vidéo apparaît à trois reprises dans ce rapport, et est perçu comme un outil permettant de changer la manière dont sont dispensés les savoirs. Tout d'abord, il est envisagé comme une opportunité pour enseigner en partant des pratiques des élèves : « Le constat que les pratiques numériques personnelles des jeunes, à travers notamment les jeux vidéo ou les réseaux sociaux, permettent de développer un certain nombre de savoir-faire, pour ne pas dire de connaissances et compétences informelles, que l'École peut (et devrait) réinvestir. $~^{13}$ Le système scolaire devrait donc les repérer, les délimiter et permettre à ses élèves de les réutiliser dans des activités scolaires. De plus, jouer permettrait de briser l'ennui de plus en plus important que les élèves ressentiraient dans la salle de classe ${ }^{14}$ : en favorisant " le plaisir et la motivation ", ce qui permettrait en retour d' " enclencher un cercle vertueux, même s'il ne garantit pas toujours de meilleurs résultats ». Dans ce cas, le jeu vidéo n'est pas vu comme un outil pour améliorer les performances scolaires, mais plutôt pour changer le climat de la classe.

Pour finir, le jeu vidéo pourrait être utilisé comme un outil de simulation :

"Le développement de la "ludification" des ressources pédagogiques comme celui des "jeux sérieux" [...] débouchent sur la mise en place de scénarios où les élèves sont amenés à vivre dans un monde virtuel, mais à comprendre et à agir comme dans la vie réelle, à partir de questions scientifiques, techniques ou de société. $»^{15}$

Le jeu vidéo est considéré comme un moyen de faire évoluer la forme scolaire. Conceptualisée par le sociologue Guy Vincent, la notion de forme scolaire rassemble différentes caractéristiques, historiquement constituées, que l'on retrouve dans le fonctionnement social et structurel de l'école : un espace dédié à l'enseignement, séparé des autres pratiques de la vie quotidienne et régis par des règles imposées, une organisation rationnelle du temps, un corpus de connaissances et de compétences à transmettre, ainsi qu'un rapport d'autorité entre les individus, ici l'enseignant et ses élèves ${ }^{16}$. Ainsi « l'école change avec le jeu numérique ", participant ainsi à un ensemble de discours voyant, en lui, un

12 Id.

13 Ibid., p. 14.

14 Caroline Brizard, "L'ennui à l'école touche tout le monde ", Nouvel Obs, 30 août 2015 : https://www.nouvelobs.com/societe/20150827.OBS4834/l-ennui-a-l-ecole-touche-tout-lemonde.html (dernière visite le 4 avril 2019).

15 Catherine Becchetti-Bizot, Repenser la forme scolaire à l'heure du numérique, op. sit., p. 46.

16 Guy Vincent, L'Éducation prisonnière de la forme scolaire? Scolarisation et socialisation dans les sociétés industrielles, Lyon, Presses universitaires de Lyon, 1994, p. 227. 
dispositif permettant de transmettre du contenu disciplinaire. Encensé par la critique, disposant d'un contenu culturel certifié par la Mission du Centenaire, et d'un contexte institutionnel favorable à son utilisation, il s'agit à présent de savoir comment Soldats Inconnus a pu être utilisé en classe.

Les enseignants sélectionnés ont été contactés suite à un appel lancé sur les réseaux sociaux Facebook et Twitter. Nos questions étaient envoyées par mail, laissant ainsi le temps à l'enseignant de répondre et de détailler sa pratique. Notre questionnaire abordait tout d'abord la culture ludique de l'enseignant, afin de déterminer si celle-ci expliquait le recours au médium vidéoludique dans sa classe. Par la suite, nous leur avons demandé de détailler leur utilisation concrète en classe. Tout d'abord, les six enseignants qui ont répondu à l'appel sont tous des joueurs de jeux vidéo. Quatre d'entre eux déclarent avoir une culture très diversifiée, sans genres de prédilection. Certains ont des pratiques de collectionneurs (Julien, enseignant en histoire), d'autres parlent volontairement de leurs autres activités ludiques comme le jeu de rôle papier (Nicolas, enseignant en histoire). En revanche, Damien, documentaliste, construit sa culture vidéoludique au contact des élèves. Il explique d'ailleurs qu' « à titre professionnel [il] joue/enseigne sur PS3, PS4 " en collaboration avec une collègue. Sa culture ludique s'imbrique donc avec sa pratique professionnelle. À noter qu'aucun des élèves de ces six enseignants ne connaissait Soldats Inconnus avant que leur enseignant ne leur fasse jouer. À partir de ce tableau de synthèse (Figure 4), nous allons présenter chacun de ces enseignants ainsi que leur séance pédagogique utilisant Soldats Inconnus.

\begin{tabular}{|c|c|c|c|c|}
\hline & Discipline & Contexte & Objectif & Posture de jeu \\
\hline Julien & Histoire & $\begin{array}{c}\text { Enseignement modulé - } \\
\text { 2nd (lycée) }\end{array}$ & $\begin{array}{c}\text { Illustrer le cours } \\
\text { d'histoire }\end{array}$ & Let's Play \\
\hline Nicolas & Histoire & $\begin{array}{l}\text { Cours d'histoire - } \\
3^{\text {e }} \text { (collège) }\end{array}$ & Séance de révision & $\begin{array}{c}\text { Jeu individuel } \\
\text { sur tablettes }\end{array}$ \\
\hline Alexis & FLE & $\begin{array}{l}\text { Elèves primo-arrivants - } \\
\text { Collège }\end{array}$ & $\begin{array}{c}\text { Travailler des } \\
\text { compétences langagières }\end{array}$ & Let's Play \\
\hline Damien & Documentation & $\begin{array}{c}\text { "Club philo"sur } \\
\text { l'heure du midi - } \\
\text { Centre de } \\
\text { Documentation } \\
\text { et d'Information (CDI) } \\
\text { (collège) }\end{array}$ & $\begin{array}{l}\text { Construire un débat } \\
\text { philosophique sur l'art } \\
\text { et la guerre }\end{array}$ & Let's play \\
\hline Thierry & $\begin{array}{l}\text { Enseignant } \\
\text { spécialisé }\end{array}$ & $\begin{array}{c}\text { Cours de Français } \\
\text { et d'histoire }\end{array}$ & $\begin{array}{c}\text { Travailler le langage } \\
\text { et les repères historiques }\end{array}$ & Let's play \\
\hline
\end{tabular}

Figure 4 : Les enseignants ayant utilisé Soldats Inconnus dans leurs cours 


\section{Julien, professeur d'histoire-géographie au lycée}

Julien utilise le jeu avec ses élèves de seconde dans le cadre des " enseignements modulés " : deux heures par semaines qui sont à disposition de l'enseignant pour mener des projets pédagogiques. Julien explique ainsi qu'il possède une certaine liberté pour aborder des points du programme avec ces heures " que l'on organise comme on veut ». Soldats Inconnus vient en complément de son cours sur l'expérience combattante et sert «à illustrer le propos et non pas le comprendre ".

Julien choisit lui-même les niveaux (La conscription, Ypres sous les attaques de gaz et Le chemin des Dames) pratiqués par un élève seul pendant que le reste de la classe regarde. Nous choisissons d'appeler cette disposition - un joueur et des spectateurs - un let's play pédagogique, terme emprunté aux vidéos YouTube où un vidéaste joue à un jeu vidéo tout en le commentant en direct.

Julien intervient dans l'activité ludique de ses élèves en leur demandant de trouver les items collectionnables, une des lacunes que nous exposions plus haut : d'objets optionnels, l'enseignant rend cette quête obligatoire. De plus, Julien oriente ensuite ses élèves vers un travail scolaire en leur demandant de rédiger des fiches sur les objets trouvés. Sa séance se termine avec un moment de débriefing, permettant aux élèves de verbaliser les aspects historiques du jeu.

\section{Nicolas, professeur d'histoire-géographie au collège}

Au collège, Nicolas a l'habitude d'utiliser des jeux dans sa pratique pédagogique. Conquis par l'aspect historique de Soldats Inconnus après l'avoir découvert à travers son réseau professionnel ("sur le papier, c'est vachement bien foutu »), Nicolas donne la possibilité à chacun de ses élèves de jouer puisqu'il dispose d'un important parc de tablettes tactiles.

Or, la séance est différente de celle de Julien puisque Nicolas utilise le jeu dans une séance de révisions. Les élèves démarraient individuellement au début du jeu et devaient répondre à une série de questions. S'ils avaient donc une liberté de jeu totale, Nicolas ne pouvant donner des directives à chaque joueur, l'enseignant estime que sa séance a échoué. Il regrette ainsi avoir donner trop d'autonomie à ses élèves, c'est à dire trop de liberté de jeu, tout en affirmant que " le gamepay ne les a pas emballés".

\section{Alexis, enseignant en Français Langue Seconde au collège}

Alexis, féru de jeux narratifs, enseigne le français à des élèves primo-arrivants, des adolescents dont le français n'est pas leur langue maternelle. Alexis profite de la grande liberté pédagogique du FLS pour utiliser régulièrement des jeux vidéo en classe. Ainsi, il se sert de Soldats Inconnus pour faire travailler les temps du passé afin d'aboutir à la rédaction d'une lettre de soldats avant son départ au front. Pour cela, le premier niveau du jeu était tout indiqué : en adoptant la posture du let's play, les élèves d'Alexis parcourent le niveau 
pour observer certains éléments de la vie de la caserne (drapeau tricolore, armement, clairon...). Les phases de jeu étaient également entrecoupées de séances en bibliothèque pour vérifier les informations historiques du jeu.

\section{Damien, documentaliste au collège}

Damien est assez critique sur Soldats Inconnus : "d'un point de vue graphique le jeu est excellent, le gameplay est appréciable, mais parfois un peu alourdi par quelques considérations historiques".

Étant documentaliste, la séance de jeu ne se déroule pas pendant un cours classique, mais pendant un club hebdomadaire "philo-jeux vidéo ", libre d'accès aux élèves. Le principe est de faire découvrir un jeu, jugé loin des pratiques habituelles des élèves et d'aboutir à une discussion « de nature philosophique ». Pendant la partie, Damien affirme que les élèves étaient libres. Or, sa description révèle les multiples interventions de l'enseignant : des informations historiques, ainsi que des aides de jeu, sont données aux élèves en cours de jeu et, surtout, le jeu est arrêté au bout d'une demi-heure afin de pouvoir atteindre l'objectif de la séance : la discussion philosophique.

\section{Thierry, enseignant dans un établissement médico-social dans un collège}

Thierry enseigne à des élèves sourds, dyslexiques ou dyspraxiques. Ainsi, le jeu vidéo est un support qu'il estime adapté à ses élèves en besoins spécifiques, car Soldats Inconnus ne limite pas l'accès au langage écrit ou oral comme pour un texte ou une vidéo. Thierry a connu Soldats Inconnus, à travers son réseau professionnel. Les objectifs de sa séance étaient de travailler les repères historiques ainsi que des compétences langagières, comme nous avons déjà pu le voir avec Alexis, enseignant de FLS.

Une nouvelle fois, un seul élève jouait et les autres observaient le déroulement du jeu. Pendant les temps libres, Thierry laissait le jeu à disposition des élèves pendant quelques minutes. La séance ludique était suivie par un débriefing permettant de raconter l'évolution de chacun des personnages du jeu. Ces échanges aboutissaient à la rédaction, par les élèves, de lettres de soldats.

\section{Synthèse}

Le jeu vidéo est un médium interdisciplinaire par excellence. Nous avons pu constater que l'histoire n'était pas la seule discipline à convoquer Soldats Inconnus, le Français et ses compétences d'oralisation ont été également sollicitées, et l'on peut supposer que la direction artistique pourrait faire l'objet d'une étude dans les disciplines artistiques (Arts Plastiques et enseignement musical). De son utilisation dans différentes disciplines, nous avons déterminé plusieurs constantes. Soldats Inconnus intervient toujours après que les notions disciplinaires ont été travaillées en cours. En effet, les élèves doivent déjà avoir 
les connaissances nécessaires pour situer l'action et comprendre le déroulé de l'histoire. Nous avons ainsi constaté que les enseignants interrogés donnaient un crédit assez faible à la capacité de Soldats Inconnus pour apprendre des savoirs déclaratifs. De fait, la séance de jeu est toujours suivie d'un instant de débriefing servant à institutionnaliser les savoirs aperçus dans le jeu. On peut donc supposer que, si apprentissage il y a, celui-ci se fait plutôt à travers ces échanges entre élèves et enseignants et non par l'activité ludique seule. Pour Audigier et Mitgutsh ${ }^{17}$, ce n'est pas le jeu qui favorise l'apprentissage scolaire en lui-même, mais l'activité qui l'entoure et, surtout, son insertion dans le processus pédagogique préparé par l'enseignant, qui revêt ici le rôle principal. Dans cette optique, Brougère cite Thiagarajan ${ }^{18}$ qui " met en évidence trois niveaux : Expérience $\rightarrow$ Réflexion $\rightarrow$ Apprentissage ». Nous entendons cet enchaînement ainsi : pour qu'il y ait apprentissage, il faut que l'expérience de jeu soit suivie d'une réflexion permettant de formaliser les connaissances tirées du jeu. Pour résumer, il faut sortir du jeu pour apprendre, mais cette extraction n'est pas sans risque sur l'activité ludique elle-même.

Le let's play était la mise en place la plus souvent rencontrée, l'enseignant misant généralement sur les réactions et discussions suscitées par la diffusion du jeu au tableau. Cette posture semble similaire à ce que Newman décrivait avec le terme de " jeu secondaire ${ }^{19}$, désignant l'engagement dans un jeu de personnes n'exerçant pas de contrôle sur le dispositif ludique. Le jeu n'engagerait donc pas que l'unique élève-joueur mais également l'ensemble de ses camarades. Il est d'ailleurs intéressant de constater que la séance où les élèves jouaient individuellement (Nicolas, enseignant d'histoire-géographie au collège) sur tablettes est celle qui semble s'être le moins bien déroulée. Nicolas était loin d'être prolixe dans la description des raisons de cet échec. On peut supposer que l'activité ludique individuelle s'est retrouvée en forte tension avec l'exigence de l'exercice de révision.

Le cas de Soldats Inconnus en classe permet d'illustrer "le paradoxe de l'enseignante $»^{20}$ abordé par Gilles Brougère, qui peut atténuer trois caractéristiques de l'activité ludique. Tout d'abord, il éliminerait l'incertitude : l'enseignant conduit ses élèves vers un objectif pédagogique donné, le jeu a donc un but précis. Ensuite, jouer en classe s'éloignerait du caractère frivole que

17 François Audigier, «La didactique des sciences sociales, de la théorie à la pratique. Les situations d'enseignement-apprentissage, liens nécessaires entre recherche et formation ", in Rosa Ávila, Alcázar Cruzet Consuelo Díez (éds.), Didáctica de las Ciencias Sociales en los nuevos planes de estudio, Jaén, 2008, p. 233-261 : http://www.ujaen.es/investiga/hum167/XIXSimposioInternacional/ download/DidacticaCienciasSocialesLibro.pdf et Konstantin Mitgutsch, "Digital play-based learning. A philosophical-pedagogical perspective on learning and playing in computer games ", in Games in Action, Gothenburg, Sweden, 2007.

18 Gilles Brougère, Jouer/apprendre, op. cit., p. 93.

19 James Newman, "The myth of the ergodic videogame ", Game studies, 2 (1), p. 1-17, 2002.

20 Gilles Brougère, Jouerlapprendre, op. cit., p. 79. 
l'on prête au jeu vidéo à cause de "l'investissement du sérieux éducatif $~^{21}$ : on ne joue pas pour jouer, on joue pour apprendre. Pour finir, la décision de jouer, centrale pour rentrer dans une activité de jeu selon Brougère, est en partie annulée par la présence de l'enseignant : c'est lui qui décide véritablement d'utiliser le jeu, et sûrement lui qui oriente les élèves-joueurs dans leurs pratiques ludiques en salle de classe.

Nous rejoignons ainsi les conclusions de Vincent Berry : «Plus on cherche à évaluer et à pédagogiser l'activité ludique, plus le jeu disparaît. À l'inverse, plus on laisse l'activité dans sa dimension ludique, moins l'apprentissage est visible $»^{22}$. Cependant, à cette méthodologie il faudrait ajouter des observations en situation afin de ne pas s'arrêter au seul discours et à la subjectivité de l'enseignant afin de cerner plus précisément l'expérience vidéoludique en classe. De plus, nous regrettons de ne pas avoir rencontré d'enseignants du primaire, classe d'âge vers lequel Soldats Inconnus semble être plus destiné.

Si Battlefield 1 n'était pas joué et illustrait ainsi la transformation du jeu vidéo en document ${ }^{23}$, Soldats Inconnus est bel et bien pratiqué par les élèves. Or, cette activité ludique est cadrée par les consignes de l'enseignant et, à chaque fois, orientée vers un travail scolaire. Plus qu'une ludification du cours d'histoire à l'aide d'un jeu vidéo, nous constatons une degamification de Soldats Inconnus, un dispositif intégrant déjà des mécaniques pédagogiques avec une interactivité limitée, comme nous l'avons vu en début d'article.

En guise d'ouverture, nous souhaitons interroger la pertinence du jeu vidéo, joué ou non, par rapport à un autre médium bien plus utilisé en classe, le cinéma, et qui entraîne un effet de réel bien plus important que les dispositifs vidéoludiques. De plus, le recours aux jeux cités dans cet article met régulièrement en avant la figure hérö̈que du soldat, notamment dans des first person shooters, comme Battlefield 1, ou même Soldats Inconnus par instants. Cette diffusion d'une valorisation de la violence et de l'acte guerrier, parfois nécessaire au plaisir ludique, doit cependant faire l'objet de la plus grande prudence pour les enseignants.

Romain Vincent

Université Paris 13

vincent.histgeo@gmail.com

21 Ibid.

22 Vincent Berry, "Jouer pour apprendre : est-ce bien sérieux ? Réflexions théoriques sur les relations entre jeu (vidéo) et apprentissage ", in Canadian Journal of Learning and Technology/ La revue canadienne de l'apprentissage et de la technologie, 37 (2), 2001.

23 Référence vers l'article de W. Brou dans l'ouvrage. 
Après l'obtention d'un master Recherche en Histoire médiévale en 2009 et du CAPES d'Histoire-Géographie en 2010, l'intérêt de Romain Vincent pour l'histoire et les jeux vidéo l'ont poussé à s'orienter vers un Master en Sciences du jeu à l'Université Paris 13 en 2017, tout en menant une activité de médiation sur la chaîne YouTube "Jeux Vidéo \& Histoire ». Après un mémoire soutenu en juillet 2018, son sujet de doctorat en sciences de l'éducation vise à poursuivre la réflexion sur l'usage pédagogique des jeux vidéo par les enseignants français : $D u$ ludique au pédagogique: ce que les enseignants font aux jeux vidéo.

\title{
Publications et communications
}

«Et si on jouait à l'Histoire? Histoire de jouer. » Intervention lors de la journée d'étude " Penser (avec) la culture vidéoludique » de l'université de Lausanne, 5 octobre 2017. (À paraître)

\section{Mémoire de master}

Romain Vincent, Jouer au jeu vidéo pour changer l'école? L'expérience vidéoludique dans le système scolaire français. Mémoire de master en sciences de l'éducation (spécialité sciences du jeu), sous la direction de Vincent Berry, Université Paris 13, 2018. Disponible sur https://dumas.ccsd. cnrs.fr/dumas-01895467.

\section{Résumé}

Cet article vise à étudier les liens entre jeux vidéo et apprentissage de l'histoire à travers Soldats Inconnus : Mémoires de la Grande Guerre d'Ubisoft, sorti en 2014. Pour cela, nous proposons deux approches. Tout d'abord, nous rendons compte de la manière dont Soldats Inconnus transmets des connaissances historiques au joueur à travers l'étude de son gameplay. Ensuite, nous décrivons comment ce jeu vidéo est utilisé dans un contexte d'apprentissage formel : la salle de classe. Ainsi, nous résumons des entretiens avec des enseignant.es de différentes disciplines et niveaux qui l'ont fait rentrer dans leur salle de classe, révélant ainsi les diverses transformations que le cadre scolaire fait subir au jeu vidéo.

\section{Mots-clés}

apprentissage, jeu vidéo, forme scolaire, grande guerre, école.

\begin{abstract}
This article aims for studying the links between video games and learning history with "Valiant Hearts ", released in 2014 by Ubisoft. Two approaches will be suggested. First, We will focus on how the game teaches historical knowledge to gamers while playing. Then, we will describe how this video game is used in a formal learning context, the classroom. Therefore, we will summarise interviews of teachers who taught different subjects and different age groups and who used the game in class. Their experiences showed a variety of transformations undergone by the video game in the school context.
\end{abstract}

\section{Keywords}

learning, video games, school context, Great War. 